\title{
TECHNIQUES FOR AUTOMATIC VIDEO CONTENT DERIVATION
}

\author{
Milan Petkovic, Vojkan Mihajlovic, Willem Jonker \\ University of Twente, The Netherlands, email: \{milan, vojkan, jonker\}@cs.utwente.nl
}

\begin{abstract}
In this paper, we focus on the use of three different techniques that support automatic derivation of video content from raw video data, namely, a spatio-temporal rule-based method, hidden Markov models, and dynamic Bayesian networks. These techniques are validated in the particular domain of tennis and Formula 1 race videos. We present the experimental results for the detection of events such as net-playing, rally, service, and forehand stroke among others in the Tennis domain, as well as excited speech, start, fly-out, passing, and highlights in the Formula 1 domain.
\end{abstract}

\section{INTRODUCTION}

As amounts of publicly available video data grow, the need to automatically infer semantics from raw video data becomes significant. However, large amounts of data and its audio-visual nature put some challenges in front of systems that manipulate this data. Problems are especially encountered with respect to content analysis and retrieval of the audio-visual data.

In this paper, we investigate the practical use of the three different techniques, namely, spatio-temporal formalization, Hidden Markov Models (HMMs), and Bayesian Networks (BNs) to solve this problem. The possible ways of the automatic extraction of semantic content from raw video data are illustrated by two case studies that are described in the paper. In the tennis case study, we demonstrate the practical exploitation of spatiotemporal reasoning and HMMs in a real situation using the ordinary TV broadcast of tennis matches. On the other hand, we analyze the use of Bayesian and dynamic Bayesian networks for multi-modal content-based video retrieval. This approach is validated in the particular domain of Formula 1 race videos for highlight extraction. Finally, the last section discusses the advantages and disadvantages of the use of the proposed techniques with respect to different kinds of events to be recognized.

\section{TENNIS CASE STUDY}

The aim of the Tennis case study is to automatically extract events like net-playing, rally, longest point, and shortest point, as well as different kinds of tennis strokes from tennis game videos. However, some preprocessing, which precedes the extraction of high-level concepts has to be done. It includes reconstruction of the video structure, i.e. the detection of shot boundaries, but also shot classification, object segmentation, and tracking. As this is out of the scope of this paper, we refer to [1] for a detailed description.

Having the video processing done and player shape segmented (Figure 1), we extract a number of features. Besides the player's position relative to the court $\left(f_{1-3}\right)$, the dominant color $\left(f_{4}\right)$, and standard shape features, such as the mass center $\left(f_{5-6}\right)$, the area $\left(f_{7}\right)$, the bounding box $\left(f_{8}\right.$ $\left.{ }_{11}\right)$, the orientation $\left(f_{12}\right)$, and the eccentricity $\left(f_{13}\right)$, we extract the following features:

The position of the upper half of the mask with respect to the mass center $\left(f_{14-15}\right)$, its orientation $\left(f_{16}\right)$, and the eccentricity $\left(f_{17}\right)$. Those features describe the upper part of the body that contains most of the information.

- For each circle cut out that is centered at the mass center, we count the number of pixels in the mask $\left(f_{18-25}\right)$. This can be seen as a general approximate description.

- The sticking-out parts $\left(f_{26-27}\right)$ are extracted by filtering and finding local maximums of the distance from a point on the contour to the mass center.

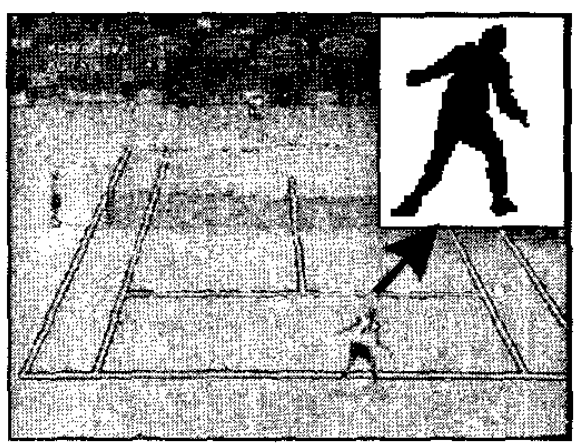

Figure 1. Segmented shape of a player

\subsection{Spatio-Temporal Event Recognition}

The spatio-temporal event formalization is used for the description of high-level concepts. To extract these concepts automatically based on features and spatiotemporal reasoning, we have developed object and event grammars that define rules for object and event descriptions based on spatial, temporal, and feature 
operators (for more details see [2]). The rules facilitate automatic mapping from features to high-level concepts (objects and events contained in a video). The syntax of rules and an engine that supports their use are described in [2]. Here, we will only give a query example from the tennis domain:

SELECT vi.frame_seq FROM video vi WHERE s_contains (vi.frame_seq, event, Player_near_the net $=\left(\left\{o_{1}:\right.\right.$ player, $o_{2}:$ net $\},\{\},\{\}$, $\left\{y_{-}\right.$distance $\left.\left(\mathrm{o}_{1}, \mathrm{o}_{2}\right)<50\right\}$, \{duration (this) $\left.>60\right\}$ ), $o_{1} \cdot$ name $=$ 'Sampras')

The query retrieves all video segments where Sampras is playing from close to the net for a given period of time. It is formulated using an extended $O Q L$, where s_contains is a function that checks if a sequence contains specific objects or events. The "player_near_the_net" event type is defined in terms of spatio-temporal object interactions. In this rule, we use a spatial relation (distance) defined on features $\mathrm{f}_{1-3}$ and also one temporal relation (duration). The temporal relation says that this event type should last a specific period, as well as that the spatial relation should be valid for that period of time. Similarly, other 'spatio-temporal' rules are used to define events like rally, lob, long point, etc [2].

Although we have demonstrated that spatio-temporal formalization can be used for inferring video semantics from low-level feature representations and extracting events like net-playing and rally, the presented approach has some drawbacks. Firstly, it is essentially restricted to the extent of recognizable events, since it might become difficult to formalize complex actions of non-rigid objects ${ }^{i}$ using the proposed approach. Especially, this holds for an ordinary user who is not familiar with video features and spatio-temporal reasoning. An expert can help, but even then for some events the approach will not grant the best results. Finally, the proposed approach requires that someone, either a user or an expert, creates object and event descriptions, which can be time-consuming and error-prone.

In order to overcome the above mentioned drawbacks, we propose the use of stochastic techniques, such as Hidden Markov Models (HMMs). These techniques exploit automatic learning capabilities to derive knowledge and avoid the need for an expert.

\subsection{Stochastic Event Recognition}

In the Tennis case study, we use Hidden Markov Models (HMMs) to recognize different strokes performed by: players, such as forehand, backhand, service, etc. Series of stroke recognition experiments are conducted to demonstrate the validity of our approach.

In our experiments, we use ordinary TV broadcast tennis videos with different players at different tournaments. For stroke recognition, we use first order, left-to-right, discrete HMMs. In order to design a codebook we use the k-means algorithm. As the selection of the codebook size is a trade-off between a smaller quantization error (larger codebook size) and faster HMM operations (smaller codebook size), we tried various codebook sizes in the range of $8-80$ symbols.

Prior to stroke recognition, we perform the learning procedure, and train the adequate number of HMMs to recognize specific classes of strokes. We set up the HMM parameters, which include the number of states and the size of a codebook, based on some heuristics. After trying different codebook sizes and number of states from 4 to 48 , we selected the codebook size of 24 symbols and HMMs with 8 states.

In the learning process, we train 50 different models for each stroke class using the Baum-Welch algorithm with the modified re-estimation formula for the training with multiple observation sequences [3]. As a final result, we select the model with the highest probability to represent a class of strokes. Having a model for each class of strokes, we perform stroke recognition.

In the experiments, we aimed at achieving of two goals: (1) determine the best feature set and (2) investigate person independence of different feature sets. Hence, we have performed a number of experiments with different feature combinations. To examine how invariant they are on different players, we used the same player in the training and evaluation sets in the first series of experiments, while in the second series HMMs were trained with one group of players, but strokes performed by another group were evaluated. In both cases, the training set contained 120 different sequences, while the evaluation set contained 240 sequences.

We selected six events to be recognized: forehand, backhand, service, smash, forehand volley, and backhand volley. In each experiment, six HMMs were constructed one for each type of events to be recognized. Each stroke sequence was evaluated by all six HMMs (parallel evaluation). The one with the highest probability was selected as the result.

The recognition accuracies (percentages of rightly classified strokes) show that the combination of pie and skeleton features $\left(f_{14-23}\right)$ achieved the highest accuracy in the experiment $1(93 \%)$. The recognition rates dropped in experiment 2 ( $87 \%$ for pie and skeleton features). Two feature combinations showed to be the most person independent, i.e. invariant on different player constitutions. The first is the combination of eccentricity, the mass center of the upper part, and skeleton features (recognition rate of $91 \%$ in the first experiment and $89 \%$ in the second), while another is the combination of orientation, eccentricity, and skeleton features $(89 \%$ and $88 \%$ ). Other feature combinations showed poorer results. 


\section{FORMULA 1 CASE STUDY}

The aim of the Formula 1 case study is twofold: Firstly, we investigate the effectiveness of Bayesian and dynamic Bayesian networks for content-based video retrieval. Secondly, we analyze the applicability of these networks for the fusion of multimodalities in the retrieval process. In particular, we center our attention on fusing the evidence obtained from different media information sources in order to extract highlights from Formula 1 race video. A detailed description of the Formula 1 case study can be found in [4].

\subsection{Information Sources}

In the process of extraction of multi-modal cues, we use three different media components of the TV broadcasting Formula 1 program: audio, video, and text.

Audio plays a significant role in the detection and recognition of events in video. In our domain, the importance of the audio signal is even bigger, since it encapsulates the announcer's comment, which can be considered as a kind of the on-line human annotation. Furthermore, whenever something important happens the announcer raises his voice due to his excitement, which is a good indication for the highlights.

Based on a few experiments, we select four audio features to be used for speech endpoint detection and extraction of excited speech. We chose Short Time Energy (STE), pitch, Mel-Frequency Cepstral Coefficients (MFCCs), and pause rate. A description of methods we developed for excited speech and speech endpoint detection can be found in [4]. For the recognition of specific keywords in announcer's speech, we used a keyword-spotting tool.

In our visual analysis, we use color, shape, and motion features. First, the video is segmented into shots. Then, we calculate the amount of motion and apply semaphore, dust, sand, and replay detectors in order to characterize passing, start, and fly-out events, as well as to find replay scenes (for a description of these detectors see [4]).

The third information source we use is the text that is superimposed on the screen. This is another type of online annotation done by the TV program producer, which brings some additional information with intention to help viewers to better understand the video content. In order to speed up the detection and recognition of the superimposed text, we modified the existing technique considering the properties of Formula 1 race videos [4].

\subsection{Probabilistic Fusion}

As the majority of techniques for event detection, which relay solely on the one-media cues, showed to have robustness problems, we decided to base our analysis on the fusion of the evidence obtained from the aforementioned information sources. In order to find the most appropriate technique, we performed numerous experiments and compare Bayesian Networks (BNs) versus Dynamic Bayesian Networks (DBNs), different network structures, temporal dependences, and learning algorithms. For learning, we employ the Expectation Maximization algorithm. In the inference process, we use the modified Boyen-Koller algorithm for approximate inference. For the descriptions of both algorithms see [4].

We digitized three Formula 1 races, namely, the German, Belgian, and USA Grand Prix (GP). Feature values, extracted from the audio and video signals, are represented as probabilistic values in range from zero to one. The features we extracted form a Formula 1 video are: keywords $\left(f_{l}\right)$, pause rate $\left(f_{2}\right)$, average values of STE $\left(f_{3}\right)$, dynamic range of STE $\left(f_{4}\right)$, maximum values of STE $\left(f_{5}\right)$, average values of pitch $\left(f_{6}\right)$, dynamic range of pitch $\left(f_{7}\right)$, maximum values of pitch $\left(f_{8}\right)$, average values of $\operatorname{MFCCs}\left(f_{9}\right)$, maximum values of MFCCs $\left(f_{10}\right)$, part of the race $\left(f_{11}\right)$, replay $\left(f_{12}\right)$, color difference $\left(f_{13}\right)$, semaphore $\left(f_{14}\right)$, dust $\left(f_{15}\right)$, sand $\left(f_{16}\right)$, and motion $\left(f_{17}\right)$.

We start our experiments by comparing the results that can be achieved by employing BNs versus DBNs for processing of only audio cues to determine exited speech. We developed three different structures of $\mathrm{BNs}$ and corresponding DBN structures. The intention was to explore how different network structures can influence the inference step in this type of networks. The structures of BNs, which are also used for one time slice of DBNs, are depicted in Figure 2.

(a)

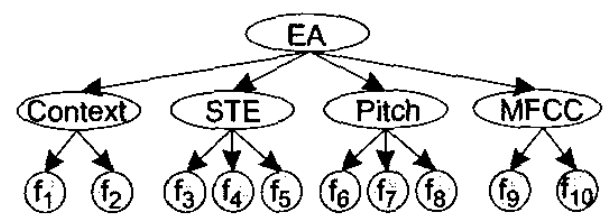

(b)

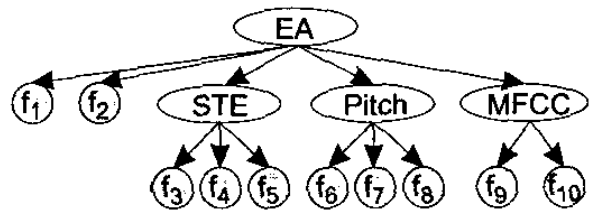

(c)

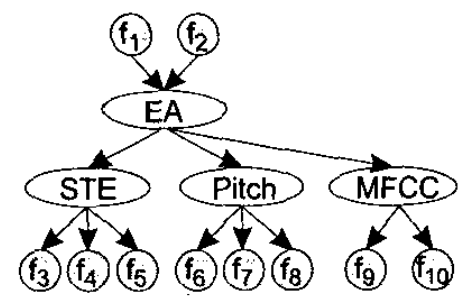

Figure 2. Different structures for processing of audio features 
The query node is Excited Announcer (EA), since we want to determine if the announcer raises his voice due to an interesting event that is taking place in the race. The shaded nodes represent evidence nodes, which receive their values based on features extracted from the audio signal of the Formula 1 video. The temporal dependencies between nodes from two consecutive time slices of DBNs were defined as in Figure 3.

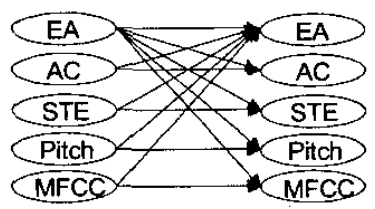

Figure 3. Temporal dependencies for the DBNs

We learned the $\mathrm{BN}$ parameters on a sequence of $300 \mathrm{~s}$, consisting of 3000 evidence values, extracted from the audio signal. For the DBNs, we used the same video sequence of $300 \mathrm{~s}$, which was divided into 12 segments with $25 \mathrm{~s}$ duration each. The inference was performed on audio evidence extracted from the German GP. For each network structure we computed precision and recall.

By comparing different $\mathrm{BN}$ structures we found that there is no significant difference in precision and recall obtained from them (around 55\%). The corresponding DBNs perform similarly, except for the DBN that corresponds to the BN depicted in Figure 2a. It gives much better results than the other BN/DBN networks (more than 80\%).

Conclusions from experiments performed are twofold. We conclude that the DBN learning and inference procedures depend a lot on the selected DBN structure for one time slice. We can see that this is not the case when inference and learning are performed with BNs. Secondly, these experiments showed the advantages ' of the DBN structure depicted in Figure 2a over the other BN/DBN networks.

The audio DBN can only extract the segments of the Formula 1 race where the announcer raises his voice. Other interesting segments (highlights), which were missed by the announcer, could not be extracted.' Therefore, the employment of the audio DBN for highlight extraction would lead to high precision, but low : recall (about $50 \%$ ).

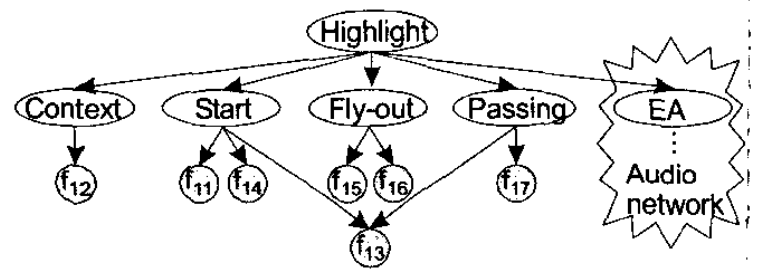

Figure 4. Audio-visual DBN for one time slice
To improve the results obtained solely from audio cues we developed an audio-visual DBN for highlight detection. The structure that represents one time slice of this network is depicted in Figure 4. The Highlight node was chosen to be the main query node, while we also queried nodes: Start, Fly Out, and Passing, in our experiments. We used the same kind of temporal dependencies as for the audio network. With this network we achieved the precision and recall rate of $80 \%$ in average for the three races.

\section{DISCUSSION}

The three approaches, which have been identified and used for bridging the semantic gap, have shown that each of them is advantageous for a specific kind of problems. The spatio-temporal formalization is beneficial for the characterization of events that have spatio-temporal nature and can be described by transitions of object positions and their spatio-temporal relations (e.g. netplaying event). HMMs and DBNs are more appropriate for events that have more 'stochastic' nature. Furthermore, by using these two techniques, we benefit of their automatic learning capabilities. Although we have not compared the two stochastic approaches between each other, our intuitive conclusion is that the DBN approach is more suitable for fusing multimodalities in retrieval. We based this conclusion on the property of DBNs that each feature can influence the decision with a specific probability. In our HMM approach the process of quantization, which leads to discrete HMMs, has treated all features equally. However, operations with HMMs are less time-consuming than with DBNs.

By integrating the work presented in this paper within the content-based video retrieval system we presented in [2], we improve the flexibility and generalize our approach, exploring the properties of databases as general purpose systems. Therefore, the necessary adjustments of the system, when the application domain changes are minimized.

\section{REFERENCES}

[1] Z. Zivkovic, et al, "Image processing and feature extraction for recognizing strokes in tennis game videos", In Proc. of ASCI, the Netherlands, 2001, pp. 262-266.

[2] M. Petkovic, Content-Based Video Retrieval Supported by Database. Technology, $\mathrm{PhD}$ Thesis, Enschede, the Netherlands, 2003.

[3] S. Michaelson, M. Steedman, Hidden Markov Models for Speech Recognition, Edinburgh University Press, 1990.

[4] V. Mihajlovic, M. Petkovic, Automatic Annotation of Formula I Races for Content-Based Video Retrieval, TRCTIT-01-41, 2001, $37 \mathrm{pp}$. 